\title{
Entanglement in Frequency Standards and Quantum Information Theory.
}

\author{
S.F. Huelga ${ }^{1}$, P.L. Knight ${ }^{1}$, C. Macchiavello ${ }^{2}$, M.B. Plenio ${ }^{1}$ and V.Vedral $^{3}$ \\ 1) Optics Section, The Blackett Laboratory, Imperial College, London SW7 2BZ, England. \\ huelga@ic.ac.uk,p.knight@ic.ac.uk and plenio@ic.ac.uk Fax: +44171 8238376, Tel: +44 1715947754 \\ 2) Dipartimento di Fisica A. Volta, Universita' di Pavia, 27100 Pavia, Italy. \\ chiara@Enterprise.pv.infn.it \\ 3) Centre for Quantum Computation, Clarendon Laboratory, Parks Road, Oxford OX1 3PU, England. \\ v.vedral1@physics.oxford.ac.uk Tel: +441865272666
}

\begin{abstract}
Entanglement is a key property of quantum information theory. Here we describe ways to quantify the amount of entanglement. We point out the statistical interpretation of these entanglement measures and some connections between entanglement transformations and thermodynamics. We also describe ways how entanglement can be applied in quantum optical applications such as optical frequency standards.
\end{abstract}

\section{INTRODUCTION}

Quantum mechanical entanglement is responsible for many strange effects in quantum theory. It allows for stronger than local correlations between the measurement results of spatially separated observers and leads for example to the violation of Bell inequalities [1]. In recent years applications of entanglement have shifted from tests of quantum mechanics towards its application in a new theory of information - quantum information theory. It has been realized that information theory is not independent of physics but is dependent on it, in the sense that physics describes the nature of the carriers of information. Classical physics forms the physical basis for classical information theory while quantum mechanics demands a quantum information theory. Many of the new effects in quantum information theory can be explained by the presence of quantum mechanical entanglement. In the first part of this article we describe some aspects of quantum entanglement and in particular consider the question of how to quantify entanglement. This allows us to uncover some deep connections between entanglement, statistics and thermodynamics.

The results of quantum information theory and in particular entanglement can also be utilized in practical applications and in this article we will consider one such example. Optical frequency standards, i.e. atomic clocks, suffer from decoherence, which ultimately limits their precision. Entanglement offers new ways to fight the effect of decoherence in frequency standards. In the second part of this article we review some of the work done in this direction. It uncovers connections between the frequency standards problem, parameter estimation and the use of partially entangled states.

\section{STATISTICAL INTERPRETATION OF THE VON NEUMANN ENTROPY}

The Shannon entropy plays a central role in classical information theory [2]. Loosely speaking it measures the amount of uncertainty in a given random variable. Suppose that a random variable $X$ has the outcomes $x_{1}, \ldots x_{n}$ appearing with the corresponding probabilities $p_{1}, \ldots p_{n}$. The Shannon entropy is then given by

$$
H(X):=-\sum_{i} p_{i} \log p_{i}
$$

The real importance of this quantity lies in the following fact. Suppose for simplicity that we have a binary source characterized by the entropy $H$, then the number of typical sequences of length $N$ generated by that source (in identical and independent fashion) is $2^{N H}$ when $N \rightarrow \infty$ [2] (this is the limit that will be assumed for the rest of this section). If the source is completely random, i.e. if 0 and 1 appear with equal probabilities, then $H=1$ and the number of sequences is the expected $2^{N}$, i.e. all sequences are equally likely. This result implies that any sequence of $\mathrm{N}$ outputs of this source can be "compressed" to the length of $N H$ (for $N$ large, of course), i.e. we are able to send a message originally consisting of $N$ bits using only $N H<N$ bits. To achieve this we just number the $2^{N H}$ most likely sequences which requires $N H$ bits. For large $N$ the probability to find a sequence that is not numbered in this way tends to zero.

Now suppose that we have two sources $X$ and $Y$ so that the total entropy is $H(X, Y)$. In this case an output sequence of length $N$ generated by these two sources can be compressed to $N H(X, Y)$. But, if we were to compress $X$ and $Y$ separately we would get the total sequence to be of the compressed length $N(H(X)+H(Y))$ (which is $\geq N H(X, Y))$. The difference between the two compressions lies in the correlations that can exist between the two source. Of course, if the two sources are correlated then by compressing one source we also compress a part of the other source (how large this part is depends on the extent of correlations). Thus a good way of quantifying these correlations is the so called Shannon mutual information [2]

$$
I(X, Y):=H(X)+H(Y)-H(X, Y) .
$$


Note that if the two sources are independent then $H(X, Y)=H(X)+H(Y)$, and the Shannon mutual information reduces to zero, indicating correctly that there are no correlations.

The above are purely classical results. The analogous results, however, can be derived for quantum information theory providing that instead of the Shannon entropy we use the von Neumann entropy, which for a system described by the density matrix $\rho$ is given by

$$
S(\rho):=-\operatorname{Tr} \rho \log \rho .
$$

The density matrix is, of course, a quantum generalisation of the probability distribution. If a quantum source producing states described by $\rho$ has an entropy $S(\rho)$, then its output string of length $N$ can be compressed to length $N S(\rho)[3]$ in direct analogy with the classical result presented above. Likewise the amount of correlations between two quantum systems in the joint state $\rho_{A B}$ is given by the von Neumann mutual information

$$
I\left(\rho_{A B}\right):=S\left(\rho_{A}\right)+S\left(\rho_{B}\right)-S\left(\rho_{A B}\right),
$$

where $\rho_{A}=\operatorname{Tr}_{B} \rho_{A B}$ is the reduced density matrix of $A$ and similarly for $B$. However, we know that two quantum systems (sources) can be correlated in a different way than two classical systems, as can be seen from Bell's inequalities. On the other hand, the above expression does not distinguish the quantum and classical contributions to the correlations. If we wish to measure only the quantum part of the correlations (called entanglement) we then need to construct a different measure. Unfortunately, as there are entangled states which do not violate Bell's inequalities [4], we cannot use Bell inequalities as the ultimate criterion either. These considerations serve as a motivation for introducing procedures that treat quantum and classical correlations differently and which therefore allow us to discriminate between them. They will in turn give rise to quantities which only measure quantum correlations (entanglement).

\section{PURIFICATION PROCEDURES}

The procedures we are about to describe in this section involve two parties, usually called Alice and Bob, who are separated by a large distance. They initially share a certain number of entangled particles, half of the particles belonging to Alice and the other half to Bob. They are allowed to manipulate their systems locally and communicate their results to each other via a classical channel. Their aim is to concentrate entanglement into a smaller subensemble of the original ensemble. In the following we will describe formally the actions that Alice and Bob are allowed to perform. This formal representation will then allow us to state general conditions that entanglement measures have to satisfy.

There are three different ingredients involved in procedures that aim to distill by local operations and classical communication a subensemble of highly entangled states from an original ensemble of less entangled states.

1. Local general measurements (LGM): The most general operation that Alice can perform is a positive operator valued measure (POVM). This can be implemented by adding a multi-level system to the particle she is holding [5]. Then she performs a joint unitary transformation on both systems. Finally she can then measure the state of the additional quantum system (see Fig. 1 for a schematical representation). Similarly Bob can perform such a POVM on his side. Any POVM acting on the state $\rho_{A B}$ that is performed in this way can be written in the form

$$
\Phi\left(\rho_{A B}\right)=\sum_{i} A_{i} \otimes B_{j} \rho_{A B} A_{i}^{\dagger} \otimes B_{j}^{\dagger},
$$

where the linear operators $A_{i}, B_{j}$ satisfy the completeness relations $\sum_{i} A_{i}^{\dagger} A_{i}=1 \mathrm{~L}$ and $\sum_{j} B_{j}^{\dagger} B_{j}=1 \mathrm{l}$.

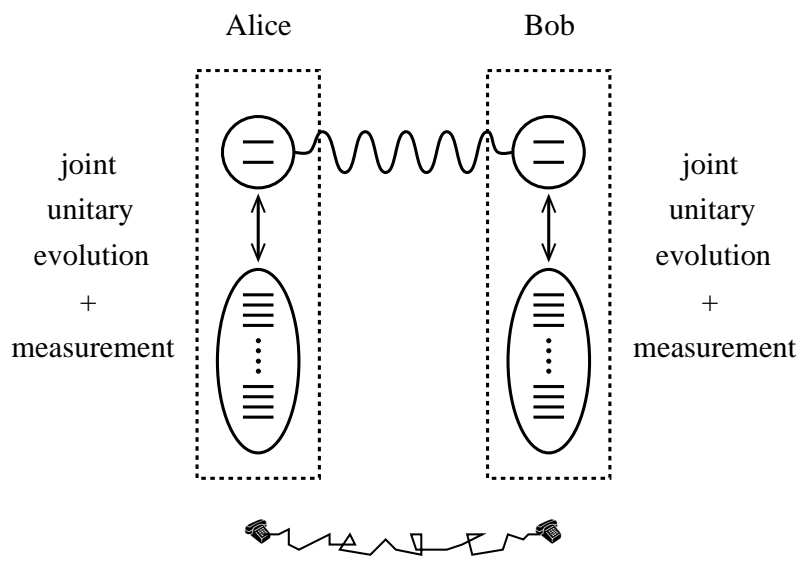

FIG. 1. Alice and Bob are allowed to perform any local general measurement. In its most general form Alice adds an additional multi-level systems to her particle and then performs a unitary transformation on the joint system followed by a measurement of the additional multi-level system. She can communicate classically with Bob about the outcome of her measurement (indicated by the telephones).

2. Classical communication (CC): Alice and Bob can choose to exchange classical information between them, e.g. the outcome of a measurement or the choice of a particular observable to be measured. This means that the actions of $A$ and $B$ can be correlated. The most general operation that Alice and Bob can perform, including classical communication, is described by a complete measurement on the whole space $A+B$ and is not necessarily decomposable into a sum of direct products of individual operators (as in LGM). If $\rho_{A B}$ describes the initial state shared between $A$ and $B$ then the transformation involving ' $\mathrm{LGM}+\mathrm{CC}$ ' would look like 


$$
\Phi\left(\rho_{A B}\right)=\sum_{i} A_{i} \otimes B_{i} \rho_{A B} A_{i}^{\dagger} \otimes B_{i}^{\dagger}
$$

where $\sum_{i} A_{i}^{\dagger} A_{i} B_{i}^{\dagger} B_{i}=1$, i.e. the actions of $A$ and $B$ are 'correlated'. Note that not all the operations of the above form can be performed locally [6]. What will for us be important is that the converse is true, namely that all the local operations and classical communication by Alice and Bob can be cast into the above form.

3. Post-selection (PS) Using classical communication Alice and Bob can chose subensembles of pairs that they are sharing (see Fig. 2). Mathematically this amounts to the general measurement not being complete, i.e. some operations are left out. The density matrix describing the newly obtained ensemble (the subensemble of the original one) has to be renormalized accordingly. Suppose that we kept only the pairs where we had an outcome corresponding to the operators $A_{i}$ and $B_{j}$, then the state of the chosen subensemble would be

$$
\rho_{A B} \longrightarrow \frac{A_{i} \otimes B_{j} \rho_{A B} A_{i}^{\dagger} \otimes B_{j}^{\dagger}}{\operatorname{Tr}\left(A_{i} \otimes B_{j} \rho_{A B} A_{i}^{\dagger} \otimes B_{j}^{\dagger}\right)}
$$

where the denominator provides the necessary normalization.

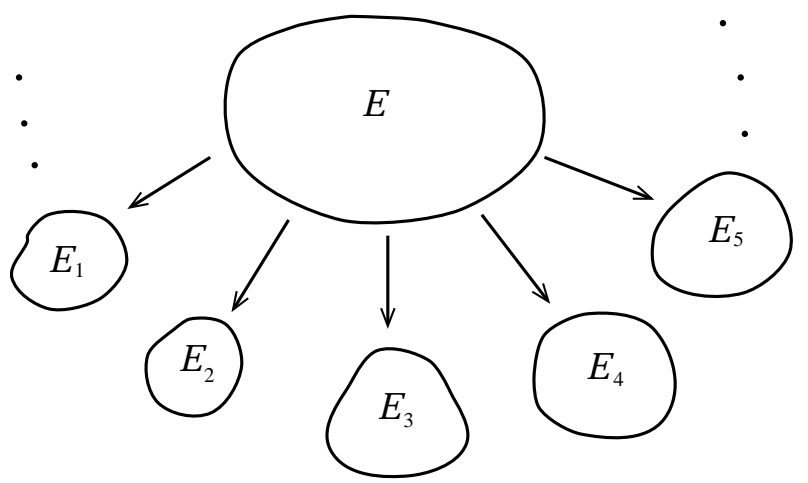

FIG. 2. Alice and Bob can communicate classically and decide to decompose their total ensemble $\mathcal{E}$ into many subensembles $\mathcal{E}_{i}$. Some of these ensembles may be described by quantum states that have a higher entanglement than the original ensemble calE.

A manipulation involving any of the above three elements or their combination we shall henceforth call an entanglement purification procedure [7]. It should be noted that the three operations described above are local. In particular, if we start with an ensemble in a product state then we will be unable to obtain a subensemble which is in an entangled state. It implies also that the entanglement of the total ensemble cannot increase under these operations (because entanglement is a non-local feature) [8]. However, classical correlations between the two subsystems can be increased, even for the whole ensemble, if we allow classical communication. A simple example confirms this. Suppose that the initial ensemble contains states $\left|0_{A}\right\rangle \otimes\left(\left|0_{B}\right\rangle+\left|1_{B}\right\rangle\right) / \sqrt{2}$. The correlations (measured by e.g. von Neumann's mutual information [9]) between A and $\mathrm{B}$ are zero. Suppose that B performs measurement of his particles in the standard 0,1 basis. If 1 is obtained, B communicates this to A who then "rotates" his qubit to the state $\left|1_{A}\right\rangle$. Otherwise they do nothing. The final state will therefore be

$\rho=\frac{1}{2}\left(\left|0_{A}\right\rangle\left\langle 0_{A}|\otimes| 0_{B}\right\rangle\left\langle 0_{B}|+| 1_{A}\right\rangle\left\langle 1_{A}|\otimes| 1_{B}\right\rangle\left\langle 1_{B}\right|\right)$,

where the correlations are now $\ln 2$ (i.e. nonzero). So, the classical content of correlations can be increased by performing local general measurements and classically communication. This provides an elegant way of distinguishing quantum from classical correlations: purification procedures can increase classical correlations, but cannot increase the total entanglement (it can only be concentrated to a smaller subensemble).

An important result was proved for pairs of spin-1/2 systems in [10]: All states that are not of the form $\rho_{A B}=\sum_{i} p_{i} \rho_{A}^{i} \otimes \rho_{B}^{i}$, where $\sum_{i} p_{i}=1$ and $p_{i} \geq 0$ for all $i$, can be distilled to a subensemble of maximally entangled states using only operations 1,2 and 3. (The states of the above form obviously remain of the same form under any purification procedure). The local nature of the above three operations implies that we define a disentangled state of two quantum systems $A$ and $B$ as a state from which by means of local operations no subensemble of entangled states can be distilled. It should be noted that these states are sometimes called separable in the existing literature. We also note that it is not proven in general that if the state is not of this form then it can be purified (in fact, the Horodeckis have recently found a counter-example to this claim in the case of two three level systems [11]). In the following we will mainly be concerned with qubits and therefore we make the following definition.

Definition 1. A state $\rho_{A B}$ is disentangled iff

$$
\rho_{A B}=\sum_{i} p_{i} \rho_{A}^{i} \otimes \rho_{B}^{i},
$$

where, as before, $\sum_{i} p_{i}=1$ and $p_{i} \geq 0$ for all $i$. Otherwise it is said to be entangled. Note that all the states in the above expansion can be taken to be pure. This is because each $\rho^{i}$ can be expanded in terms of its eigenvectors. So, in the above sum we can in addition require that $\rho_{A}^{i}{ }^{2}=\rho_{A}^{i}$ and $\rho_{B}^{i}{ }^{2}=\rho_{B}^{i}$ for all $i$ and the number of terms in the summation can be limited to the product of the dimensions of $A$ and $B$ [12] (this follows from Caratheodory's theorem [13]). 


\section{QUANTIFICATION OF ENTANGLEMENT}

In the previous section we have indicated that out of certain states it is possible to distill by means of $\mathrm{LGM}+\mathrm{CC}+\mathrm{PS}$ a subensemble of maximally entangled states (we call these states entangled). The question remains open about how much entanglement a certain state contains. Of course, this question is not entirely well defined unless we state what physical circumstances characterize the amount of entanglement. One may ask for the amount of entanglement that can be distilled from a given ensemble but one can also ask for the amount of entanglement that needs to be invested to create a given ensemble by local operations. This suggests that there is no unique measure of entanglement. Before we define some possible measures of entanglement we state three conditions that every measure of entanglement has to satisfy [12].

\section{E1. $E(\sigma)=0$ iff $\sigma$ is separable.}

E2. Local unitary operations leave $E(\sigma)$ invariant, i.e. $E(\sigma)=E\left(U_{A} \otimes U_{B} \sigma U_{A}^{\dagger} \otimes U_{B}^{\dagger}\right)$, where $U_{A}, U_{B}$ are unitary operators.

E3. The expected entanglement cannot increase under LGM+CC+PS given by operators $V_{i}$ satisfying the normalization condition $\sum V_{i}^{\dagger} V_{i}=1$, i.e.

$$
\sum \operatorname{tr}\left(\sigma_{i}\right) E\left(\sigma_{i} / \operatorname{tr}\left(\sigma_{i}\right)\right) \leq E(\sigma)
$$

where $\sigma_{i}=V_{i} \sigma V_{i}^{\dagger}$.

Condition E1 ensures that disentangled (and only disentangled states) have a zero value of entanglement. Condition E2 ensures that a local change of basis has no effect on the amount of entanglement. Condition E3 is intended to remove the possibility of increasing entanglement by performing local measurements aided by classical communication. Condition E3 takes into account the fact that we have some knowledge of the final state. Namely, when we start with $n$ systems all in the state $\sigma$ we know exactly which $m_{i}=n \times \operatorname{tr}\left(\sigma_{i}\right)$ pairs will end up in the state $\sigma_{i}$ after performing a purification procedure. Therefore we can separately access the entanglement in each of the possible subensembles described by $\sigma_{i}$. Clearly the total expected entanglement at the end should not exceed the original entanglement, which is stated by E3. This, of course, does not exclude the possibility that we can select a subensemble whose entanglement per pair is higher than the original entanglement per pair. We now introduce three different measures of entanglement which obey E1-E3 [12].

First we discuss the entanglement of formation [14]. Bennett et al [14] define the entanglement of formation of a state $\rho$ by

$$
E_{c}(\rho):=\min \sum_{i} p_{i} S\left(\rho_{A}^{i}\right)
$$

where $S\left(\rho_{A}\right)=-\operatorname{tr} \rho_{A} \ln \rho_{A}$ is the von Neumann entropy and the minimum is taken over all the possible realisations of the state, $\rho_{A B}=\sum_{j} p_{j}\left|\psi_{j}\right\rangle\left\langle\psi_{j}\right|$ with $\rho_{A}^{i}=\operatorname{tr}_{B}\left(\left|\psi_{i}\right\rangle\left\langle\psi_{i}\right|\right)$. The entanglement of formation satisfies all the three conditions E1-E3 [14]. The physical basis of this measure presents the number of singlets needed to be shared by Alice and Bob in order for them to create a given entangled state by local operations. The entanglement of formation has a number of nice properties and in particular for two spin $1 / 2$ systems a closed form of the entanglement of formation has been proven [15].

Related to this measure is the entanglement of distillation [14]. It defines the amount of entanglement of a state $\sigma$ as the proportion of singlets that can be distilled using a purification procedure (Bennett et al distinguish one and two way communication which give rise to two different measures, but we will not go into that much detail; we assume the most general two way communication). As such, it is dependent on the efficiency of a particular purification procedure and can be made more general only by introducing some sort of universal purification procedure or asking for the best state dependent purification procedure. An interesting question is also whether the entanglement of formation and the entanglement of distillation are equal. Indeed for pure states of two qubits this is the case [7]. For mixed states, however, this question is quite difficult to answer. In the following we will briefly describe a third measure of entanglement that will help us to answer this question $[12,9,16]$.

If $\mathcal{D}$ is the set of all disentangled states, the measure of entanglement for a state $\sigma$ is then defined as

$$
E(\sigma):=\min _{\rho \in \mathcal{D}} D(\sigma \| \rho)
$$

where $D$ is any measure of distance (not necessarily a metric) between the two density matrices $\rho$ and $\sigma$ such that $E(\sigma)$ satisfies the above three conditions E1-E3 (see Fig. 3). 


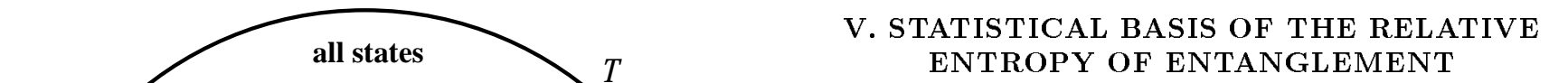

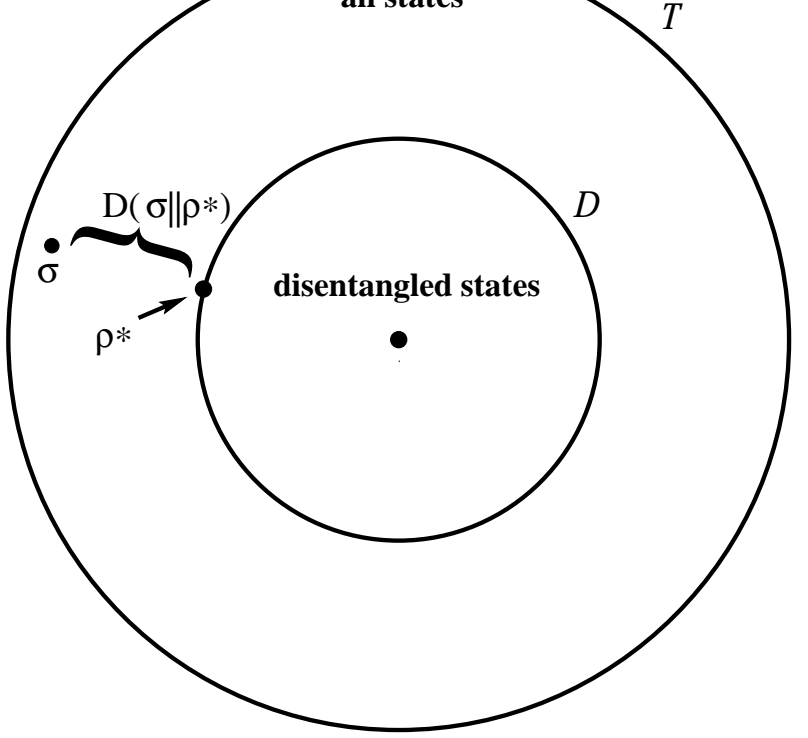

FIG. 3. A geometric way to quantify entanglement. The set of all density matrices $\mathcal{T}$ is represented by the outer circle. Its subset of disentangled (separable) states $\mathcal{D}$, is represented by the inner circle. A state $\sigma$ belongs to the entangled states, and $\rho^{*}$ is the disentangled state that minimizes the distance $D(\sigma \| \rho)$. This minimal distance can be defined as the amount of entanglement in $\sigma$.

Now the central question is what are the candidates for $D(\sigma \| \rho)$. There are many candidates for this measure $[9,12]$, but for our purposes the most useful one is the Quantum Relative Entropy given by

$$
S(\sigma \| \rho):=\operatorname{tr}(\sigma \ln \sigma-\sigma \ln \rho) .
$$

When this is used as $D(\sigma \| \rho)$ the resulting measure of entanglement will be called the Relative Entropy of Entanglement. The Relative Entropy of Entanglement satisfies the physically intuitive conditions E1-E3 (for proofs see [12]). In addition it can be shown that the Relative Entropy of Entanglement is never larger than the entanglement of formation, and in fact for Werner states the Relative Entropy of Entanglement is much smaller than the entanglement of formation. From condition E3 it follows that both the Relative Entropy of Entanglement and the entanglement of formation are upper bounds on the distillable entanglement. As the Relative Entropy of Entanglement is smaller than the entropy of formation it is therefore clear that the entanglement of distillation is smaller than the entanglement of formation. This means that usually in the generation of a mixed entangled state some entanglement is lost irreversibly.

In addition to this result that one can derive from the Relative Entropy of Entanglement one should note that this measure has an attractive statistical interpretation which offers a radically new way of looking at entanglement.
Let us see how we can interpret our entanglement measure in the light of experiments, i.e. statistically. This viewpoint is presented in greater detail in [16]. Here we present a summary sufficient to understand the ideas developed in the following section. Our interpretation relies on the result concerning the asymptotics of the Quantum Relative Entropy first proved in [17], and here presented under the name of Quantum Sanov Theorem. We first show how the notion of Relative Entropy arises in classical information theory as a measure of distinguishability of two probability distributions. We then generalize this idea to the quantum case, i.e. to distinguish between two quantum states. We will see that this naturally leads to the notion of the Quantum Relative Entropy. It is then straightforward to extend this concept to explain the Relative Entropy of Entanglement. Suppose we would like to check if a given coin is "fair", i.e. if it generates a "headtail" distribution of $\left\{p_{f}\right\}=\{1 / 2,1 / 2\}$. If the coin is biased then it will produce some other "unfair" distribution, say $\left\{p_{u f}\right\}=\{1 / 3,2 / 3\}$. So, our question of the coin fairness boils down to how well we can differentiate between two given probability distributions given a finite, $n$, number of experiments to perform on one of the two distributions. In the case of a coin we would toss it $n$ times and record the number of 0's and 1's. From simple statistics we know that if the coin is fair then the number of 0 's $N(0)$ will be roughly $n / 2-\sqrt{n} \leq N(0) \leq n / 2+\sqrt{n}$, for large $n$ and the same for the number of 1's. So if our experimentally determined values do not fall within the above limits the coin is not fair. We can look at this from another point of view: Namely, what is the probability that a fair coin will be mistaken for an unfair one with the distribution of $\{1 / 3,2 / 3\}$ given $n$ trials on the fair coin? For large $n$ the answer is $[2,12,16]$

$$
p(\text { fair } \rightarrow \text { unfair })=e^{-n S_{c l}(u f|| f)},
$$

where $S_{c l}\left(\left\{p_{u f}\right\} \|\left\{p_{f}\right\}\right)=1 / 3 \ln 1 / 3+2 / 3 \ln 2 / 3-$ $1 / 3 \ln 1 / 2-2 / 3 \ln 1 / 2$ is the Classical Relative Entropy for the two distributions. So,

$$
p(\text { fair } \rightarrow \text { unfair })=3^{n} 2^{-\frac{5}{3} n},
$$

which tends exponentially to zero with $n \rightarrow \infty$. In fact we see that already after $\sim 20$ trials the probability of mistaking the two distributions is vanishingly small, $\leq$ $10^{-10}$

This result is true, in general, for any two distributions. Asymptotically the probability of not distinguishing the distributions $P(x)$ and $Q(x)$ after $n$ trials is $e^{-n S_{c l}(P(x) \| Q(x))}$, where

$$
S_{c l}(P(x) \| Q(x))=\sum_{i} p_{i} \ln p_{i}-p_{i} \ln q_{i}
$$

(this statement is sometimes called Sanov's theorem [2]). To generalize this to quantum theory, we need a means of 
generating probability distributions from two quantum states $\sigma$ and $\rho$. This is accomplished by introducing a general measurement $E_{i}^{\dagger} \sum_{i} E_{i}=11$. So, the probabilities are given by

$$
\begin{aligned}
& p_{i}=\operatorname{tr}\left(E_{i}^{\dagger} E_{i} \rho\right) \\
& q_{i}=\operatorname{tr}\left(E_{i}^{\dagger} E_{i} \sigma\right) .
\end{aligned}
$$

Now, we can use eq. (16) to distinguish between $\sigma$ and $\rho$. The above is not the most general measurement that we can make, however. In general we have $N$ copies of $\sigma$ and $\rho$ in the state

$$
\begin{aligned}
\sigma^{N} & =\underbrace{\sigma \otimes \sigma \ldots \otimes \sigma}_{\text {total of N terms }} \\
\rho^{N} & =\underbrace{\rho \otimes \rho \ldots \otimes \rho}_{\text {total of N terms }}
\end{aligned}
$$

We may now apply a POVM $\sum_{i} A_{i}=11$ acting on $\sigma^{N}$ and $\rho^{N}$. Consequently, we define a new type of relative entropy

$$
\begin{aligned}
S_{N}(\sigma \| \rho) & :=\sup _{\mathrm{A}}{ }^{\prime}\left\{\frac{1}{N} \sum_{i} \operatorname{tr} A_{i} \sigma^{N} \ln \operatorname{tr} A_{i} \sigma^{N}\right. \\
& \left.-\operatorname{tr} A_{i} \sigma^{N} \ln \operatorname{tr} A_{i} \rho^{N}\right\}
\end{aligned}
$$

Now it can be shown that [19]

$$
S(\sigma \| \rho) \geq S_{N}
$$

where, as before,

$$
S(\sigma \| \rho):=\operatorname{tr}(\sigma \ln \sigma-\sigma \ln \rho)
$$

is the Quantum Relative Entropy [9,16,18-20]. Equality is achieved in eq. (21) iff $\sigma$ and $\rho$ commute [21]. However, for any $\sigma$ and $\rho$ it is true that $[17]$

$$
S(\sigma \| \rho)=\lim _{N \rightarrow \infty} S_{N}
$$

In fact, this limit can be achieved by projective measurements which are independent of $\sigma$ [22]. It is known that if eq. (16) is maximized over all general measurements $E$, the upper bound is given by the Quantum Relative Entropy (see e.g. [19]). In quantum theory we therefore state a law analogous to Sanov's theorem (see also [16]), Theorem 1 (or The Quantum Sanov Theorem). The probability of not distinguishing two quantum states (i.e. density matrices) $\sigma$ and $\rho$ after $n$ measurements is

$$
p(\rho \rightarrow \sigma)=e^{-n S(\sigma \| \rho)}
$$

In fact, as explained before, this bound is reached asymptotically [17], and the measurements achieving this are global projectors independent of the state $\sigma$ [22]. We note that the Quantum Sanov Theorem was presented by Donald in [23] as a definition justified by properties uniquely characterizing the quantity $e^{-n S(\sigma \| \rho)}$. The underlying intuition in the above measurement approach and Donald's approach are basically the same. Now the interpretation of the Relative Entropy of Entanglement becomes immediately transparent [16]. The probability of mistaking an entangled state $\sigma$ for a closest, disentangled state, $\rho$, is $e^{-n \times \min _{\rho} \in \mathcal{D} S(\sigma, \rho)}=e^{-n E(\sigma)}$. If the amount of entanglement of $\sigma$ is greater, then it takes fewer measurements to distinguish it from a disentangled state (or, fixing $\mathrm{n}$, there is a smaller probability of confusing it with some disentangled state). Let us give an example. Consider a state $(|00\rangle+|11\rangle) / \sqrt{2}$, known to be a maximally entangled state. The closest to it is the disentangled state $(|00\rangle\langle 00|+| 11\rangle\langle 11|) / 2[9]$. To distinguish these states it is enough to perform projections onto $(|00\rangle+|11\rangle) / \sqrt{2}$. If the state that we are measuring is the above mixture, then the sequence of results (1 for a successful projection, and 0 for an unsuccessful projection) will contain on average an equal number of 0 's and 1 's. For this to be mistaken for the above pure state the sequence has to contain all $n 1$ 's. The probability for that is $2^{-n}$, which also comes from using eq. (23). If, on the other hand, we performed projections onto the pure state itself, we would then never confuse it with a mixture, and from eq. (23) the probability is seen to be $e^{-\infty}=0$. We next apply this simple idea to obtaining an upper bound to the efficiency of any purification procedure.

\section{DISTINGUISHABILITY AND PURIFICATION PROCEDURES}

There are two ways to produce an upper bound to the efficiency of any purification procedure. Using condition E3 and the fact that the Relative Entropy of Entanglement is additive, we can immediately derive this bound. However, the additivity of the Relative Entropy of Entanglement is only a well supported conjecture at present [12] (additivity states that the entanglement of two independent entangled systems is equal to the sum of the individual amounts of entanglement). On the other hand, this upper bound on distillable entanglement can be derived in an entirely different way (but which still depends implicitly on the additivity property). This time we abandon conditions E1-E3 and use only the statistical ideas of the previous section to put an upper bound to the efficiency of purification procedures. In particular, using this new viewpoint, we show that the entanglement of formation is in general larger than the entanglement of distillation. This is in contrast with the situation for pure states where these quantities coincide [7]. The Quantum Relative Entropy is seen to play a distinctive role here, and is singled out as a 'good' generator of a measure of entanglement from among other candidates [12] which is why we have used it throughout this paper. In the previous section we presented a statistical basis to the Relative Entropy of Entanglement by considering distin- 
guishability of two (or more) quantum states encapsulated in the form of the Quantum Sanov Theorem. We now use this Quantum Sanov Theorem to put an upper bound on the amount of entanglement that can be distilled using any purification procedure. This line of reasoning follows from the fact that any purification scheme can be viewed as a measurement to distinguish entangled and disentangled quantum states. Suppose that there exists a purification procedure with the following property

- Initially there are $n$ copies of the state $\sigma$. If $\sigma$ is entangled, then the end product is $0<m \leq n$ singlets and $n-m$ states in $\rho \in \mathcal{D}$. Otherwise, the final state does not contain any entanglement, i.e. $m=0$ (in fact, there is nothing special about singlets: the final state can be any other known, maximally entangled state because these can be converted into singlets by applying local unitary operations).

Note that we can allow the complete knowledge of the state $\sigma$. We also allow that purification procedures differ for different states $\sigma$. Perhaps there is a "universal" purification procedure independent of the initial state, but presently known procedures either require knowledge of the initial states [10] or they cannot purify all disentangled states [7]. The above is therefore an idealization that may never be achieved. Now, by calculating the upper bound on the efficiency of the procedure described above we present an absolute bound for any particular procedure. We ask: "What is the largest number of singlets that can be produced (distilled) from $n$ pairs in state $\sigma$ "? Suppose that we produce $m$ pairs. We now project them non-locally onto the singlet state. The procedure will yield positive outcomes (1) with certainty so long as the state we measure indeed is a singlet. Suppose that after performing singlet projections onto all $\mathrm{m}$ particles we get a string of $m$ 1's. From this we conclude that the final state is a singlet (and therefore the initial state $\sigma$ was entangled). However, we could have made a mistake. But with what probability? The answer is as follows: the largest probability of making a wrong inference is $2^{-m}=e^{-m \ln 2}$ (if the state that we were measuring had an overlap with a singlet state of $1 / 2$ ). On the other hand, if we were measuring $\sigma$ from the very beginning (without performing the purification first), then the probability (i.e. the lower bound) of the wrong inference would be $e^{-n E(\sigma)}$. But, the purification procedure might waste some information (i.e. it is just a particular way of distinguishing entangled from disentangled states, not necessarily the best one), so that the following has to hold

$$
e^{-n E(\sigma)} \leq e^{-m \ln 2}
$$

which implies that

$$
n E(\sigma) \geq m,
$$

i.e. we cannot obtain more entanglement than is originally present. This, of course, is also directly guaranteed by our condition E3. The above, however, was a deliberate exercise in deriving the same result from a different perspective, abandoning conditions E1-E3. Therefore the measure of entanglement given in eq. (12), when $D(\sigma \| \rho)=S(\sigma \| \rho)$, can be used to provide an upper bound on the efficiency of any purification procedure. For Bell diagonal states, Rains [24] has found an upper bound on distillable entanglement using completely different methods. It turns out that the bound that he obtains in this case is identical to the one provided by the relative entropy of entanglement.

Actually, in the above considerations we have implicitly assumed that the entanglement of $n$ pairs, equivalently prepared in the state $\sigma$, is the same as $n \times E(\sigma)$. We already have indicated that this is a conjecture with a strongly supported basis in the case of the Quantum Relative Entropy (see [12]). Based on the upper bound considerations we can introduce the following definition. Definition 2. A purification procedure given by a local complete positive trace preserving map $\sigma \rightarrow \sum V_{i} \sigma V_{i}^{\dagger}$ is defined to be ideal in terms of efficiency iff

$$
\sum \operatorname{tr}\left(\sigma_{i}\right) E\left(\sigma_{i} / \operatorname{tr}\left(\sigma_{i}\right)\right)=E(\sigma)
$$

where, as usual, $\sigma_{i}=V_{i} \sigma V_{i}^{\dagger}$ and $p_{i}=\operatorname{tr}\left(V_{i} \sigma V_{i}^{\dagger}\right)$ (i.e. a the ideal purification is the one where E3 is an equality rather than an inequality). Notice an apparent formal analogy between the purification procedure and the Carnot cycle in Thermodynamics (this connection between entanglement transformations and thermodynamics is presented in more detail in [8]). The Carnot cycle is the most efficient cycle in Thermodynamics (i.e. it yields the greatest "useful work to heat" ratio), since it is reversible (i.e. it conserves the thermodynamical entropy). We would now like to claim that the ideal purification procedure is the most efficient purification procedure (i.e. it yields the greatest number of singlets for a given input state), if it is reversible (i.e. it conserves entanglement, measured by the minimum of the Quantum Relative Entropy over all disentangled states). Unfortunately this analogy between the Carnot cycle and purification procedures is not exact (it is only strictly true for the pure states). This is seen when we compare the entanglement of formation with the Relative Entropy of Entanglement. As already mentioned earlier it turns out that the entanglement of formation is never smaller than the Relative Entropy of Entanglement. This implies that the entanglement of distillation, which is bounded from above by the Relative Entropy of Entanglement, will usually be different from the entanglement of formation. This result leads to the following

Implication. In general, the amount of entanglement that was initially invested in the creation of $\sigma$ cannot all be recovered ("distilled") by local purification procedures.

Therefore, the ideal purification procedure, though most efficient, is nevertheless irreversible, and some of the invested entanglement is lost in the purification process 
itself. The solution to this irreversibility lies in the loss of certain information as can easily be seen from the following analysis. Suppose we start with an ensemble of $N$ singlets and we want to locally create any mixed state $\sigma$. Now $\sigma$ can always be written as a mixture of pure states $\Psi_{1}, \Psi_{2}, \ldots$ with the corresponding probabilities $p_{1}, p_{2}, \ldots$ We now use Bennett et al's (de)purification procedure [7] for pure states (whose efficiency is governed by the von Neumann entropy). We convert the first $p_{1} \times N$ singlets into the state $\Psi_{1}$, second $p_{2} \times N$ singlets into the state $\Psi_{2}$, and so on... In this way, the whole ensemble is in the state $\sigma$. But, we have an additional information: we know exactly that the first $p_{1} \times N$ pairs are in the state $\Psi_{1}$, the second $p_{2} \times N$ states are in the state $\Psi_{2}$, and so on. This is not the same as being given an initial ensemble of identically prepared pairs in the state $\sigma$ without any additional information. In this, second, case we do not have the additional information of knowing exactly the state of each of the pairs. This is why purification without this knowledge is less efficient, and hence one expects that the Relative Entropy of Entanglement is smaller than the entanglement of formation.

\section{ENTANGLEMENT IN FREQUENCY STANDARDS}

The aim of a frequency standard is to stabilize the frequency of a reference oscillator to a given atomic transition frequency. The development of laser cooling and trapping techniques has allowed new possibilities for improved frequency standards based on laser cooled ions. Limitations due to the second order Doppler effect present in traditional atomic beam devices can now be overcome and accuracies in the range of 1 part in $10^{14}-10^{18}$ seem achievable.

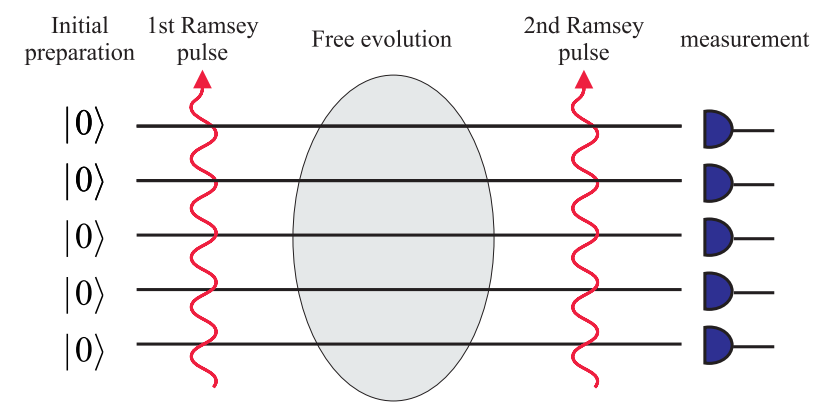

FIG. 4. Schematic representation of Ramsey-type spectroscopy with uncorrelated particles.

Let us first briefly review the implementation of a frequency standard in an ion trap, using the method of separated oscillatory fields due to Ramsey [25], illustrated in Figure 1. The ion trap is loaded with $n$ ions initially prepared in the same internal state $|0\rangle$ (we denote by $|0\rangle$ and $|1\rangle$ the ground and the excited states of each ion).
Thus the global initial state of the $n$ ions can be written as a product state of the form

$$
\left|\Psi_{n}\right\rangle=|0\rangle \otimes|0\rangle \otimes \ldots \otimes|0\rangle .
$$

A Ramsey pulse of frequency $\omega$ is applied to all ions. The frequency $\omega$ is tuned to drive the atomic transition $|0\rangle \leftrightarrow|1\rangle$ of resonance frequency $\omega_{0}$, while the pulse shape and duration are carefully chosen to prepare an equally weighted superposition of the two internal states $|0\rangle$ and $|1\rangle$ for each ion,

$$
|0\rangle \rightarrow \frac{|0\rangle+|1\rangle}{\sqrt{2}}, \quad|1\rangle \rightarrow \frac{-|0\rangle+|1\rangle}{\sqrt{2}} .
$$

Here we actually assumed that the Rabi frequency of the local oscillator is much larger than the detuning $\Delta=$ $\omega-\omega_{0}$ between the classical driving field and the atomic transition. Next the system evolves freely for a time $t$. This evolution can be expressed as a conditional phase shift on the basis atomic states:

$$
|0\rangle \rightarrow|0\rangle, \quad|1\rangle \rightarrow e^{i \Delta t}|1\rangle
$$

as can be easily seen when writing the Hamilton operator in a reference frame rotating at the oscillator frequency $\omega$ :

$$
H=-\hbar \Delta|1\rangle\langle 1| \text {. }
$$

Therefore, the frequency difference between the atomic transition and the reference oscillator leads to the accumulation of a relative phase proportional to the detuning $\Delta$. The purpose of the scheme is then to estimate the value of such detuning with the best possible precision.

The following step is to apply a second Ramsey pulse of the same characteristics as the first one. The probability that an ion is found in the state $|1\rangle$ after this pulse is given by

$$
P=(1+\cos \Delta t) / 2 \text {. }
$$

When this basic scheme is repeated yielding a total duration $T$ of the experiment, the resulting curve for the measured population in the excited state allows us to estimate the detuning and subsequently to adjust the frequency of the reference oscillator accordingly. We will now investigate the following question. Given a total duration for our experiment, $T$, and a fixed given number of ions $n$, what is the ultimate limit to the resolution of our frequency standard? Or, in other words, what is the best precision that can be achieved in the measurement of the atomic frequency?

The statistical fluctuations associated with a finite (but large) number of measurements $N$ yield an uncertainty $\Delta P$ in the estimated value of $P$ given by

$$
\Delta P=\sqrt{P(1-P) / N},
$$

where $N=n T / t$. Hence the uncertainty in the estimated value of $\omega_{0}$ is given by: 


$$
\left|\delta \omega_{0}\right|=\frac{\sqrt{P(1-P) / N}}{|d P / d \omega|}=\frac{1}{\sqrt{n T t}}
$$

This value is known as the shot noise limit [26]. We should stress that this limit arises from the intrinsic statistical character of Quantum Mechanics, in contrast to other possible sources of technical noise. While the latter may eventually be reduced, or even suppressed, the shot noise poses a fundamental limit to the achievable resolution in precision spectroscopy with $n$ independent particles.

However, the shot noise can be overcome by choosing entangled initial preparations for the state of the $n$ ions $[27,28]$. Let us consider the case where $n$ ions are prepared in the maximally entangled state

$$
\left|\Psi_{n}\right\rangle=(|00 \ldots 0\rangle+|11 \ldots 1\rangle) / \sqrt{2}
$$

If we start with all ions initially prepared in the ground state $|0\rangle$, the entangled state (34) can be generated, for example, by applying a Ramsey pulse to the first ion followed by a sequence of $n-1$ Controlled-NOT quantum gates linking the first ion with each of the remaining ones. In this paper we assume that the Controlled-NOT operations are perfect. Like in the previous method, the $n$ ions in state (34) are now allowed to evolve freely for a time interval $t$. After the free evolution period the global state in the interaction picture rotating at the driving frequency $\omega$ reads

$$
\left|\Psi_{n}\right\rangle=\left(|00 \ldots 0\rangle+e^{n i \Delta t}|11 \ldots 1\rangle\right) / \sqrt{2} .
$$

The same quantum network composed of a cascade of $n-1$ Controlled-NOT gates is now applied again to disentangle the (n-1)-ions from the first one. Finally, the probability of finding the first ion in state $|1\rangle$ is measured. It takes the form

$$
P_{n}=(1+\cos n \Delta t) / 2,
$$

namely the oscillation frequency of the signal is now amplified by a factor $n$ with respect to the case of uncorrelated ions. The corresponding frequency uncertainty reads

$$
\left|\delta \omega_{0}\right|=\frac{1}{n \sqrt{T t}} .
$$

Therefore, the use of a maximally entangled state of $n$ ions allows for an improvement of a factor $1 / \sqrt{n}$ over the shot noise limit (33) in an experiment of total duration $T$.

The remaining of this section will be devoted to an analysis of whether this improvement holds when a realistic experimental scenario is considered.

The main source of decoherence in an ion trap is of the dephasing type. This is due to processes that cause random changes in the relative phase of quantum states while preserving the population in the atomic levels. Important mechanisms that result in dephasing effects (errors) are collisions, stray fields and instabilities of the local oscillator. The free evolution period can be thought as a dephasing channel causing the initial pure state $\left|\Psi_{n}\right\rangle$ of the $n$ ions evolve into a mixed state. Assuming that phase errors occur independently, we model the time evolution of the reduced density operator for a single ion $\rho$ in the presence of decoherence by the following master equation [29]:

$$
\frac{\partial \rho}{\partial t}=-i \Delta(\rho|1\rangle\langle 1|-| 1\rangle\langle 1| \rho)+\gamma\left(\sigma_{z} \rho \sigma_{z}-\rho\right)
$$

Equation (38) is written in a reference frame rotating at the frequency $\omega$. By $\sigma_{z}=|0\rangle\langle 0|-| 1\rangle\langle 1|$ we denote a Pauli spin operator. Here we have introduced the decay rate $\gamma=1 / \tau_{\text {dec }}$, where $\tau_{\text {dec }}$ is the decoherence time. For the case of independent particles this will give rise to a broadening of the signal (31):

$$
P=\left(1+\cos \Delta t e^{-\gamma t}\right) / 2 \text {. }
$$

As a consequence the corresponding uncertainty in the atomic frequency is no longer detuning-independent. We now have

$$
\left|\delta \omega_{0}\right|=\sqrt{\frac{1-\cos ^{2}(\Delta t) e^{-2 \gamma t}}{n T t e^{-2 \gamma t} \sin ^{2}(\Delta t)}}
$$

In order to obtain the best precision it is necessary to optimise this expression as a function of the duration of each single measurement $t$. The minimal value is attained for

$$
\Delta t=k \pi / 2\left(\begin{array}{ll}
k & o d d
\end{array}\right) \quad t=\tau_{\text {dec }} / 2
$$

provided that $T>\tau_{d e c} / 2$, as it is required for high precision experiments. Thus the minimum frequency uncertainty reads

$$
\left|\delta \omega_{0}\right|_{\text {opt }}=\sqrt{\frac{2 \gamma e}{n T}}=\sqrt{\frac{2 e}{n \tau_{d e c} T}} .
$$

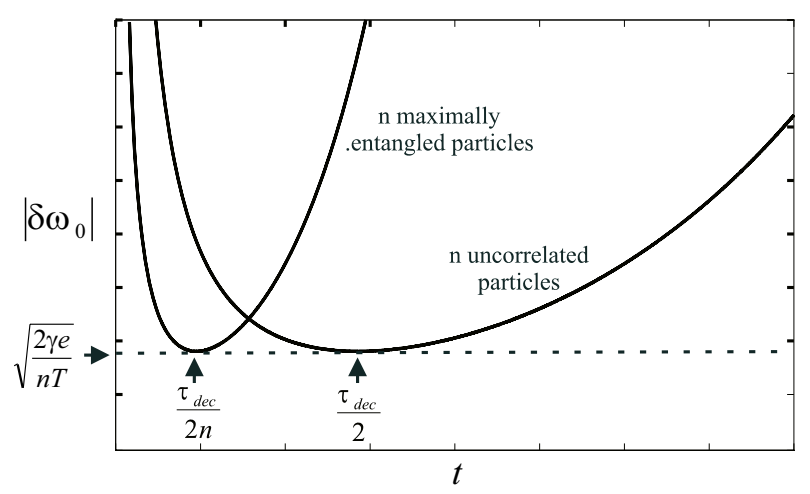


FIG. 5. Frequency uncertainty $\left|\delta \omega_{0}\right|$ as a function of the duration of a single shot $t$ for maximally entangled and uncorrelated particles. The total duration of the experiment $T$ is assumed to be larger than the characteristic decoherence time.

For maximally entangled preparation the signal (36) in the presence of dephasing is modified as follows:

$$
P_{n}=\left(1+\cos n \Delta t e^{-n \gamma t}\right) / 2,
$$

namely the oscillating term picks up a damping factor which is also amplified by $n$ with respect to the independent ions' signal (39). The resulting uncertainty for the estimated value of the atomic frequency is now minimal when

$$
\Delta t=k \pi / 2 n(k \text { odd }) \quad t=\tau_{\text {dec }} / 2 n .
$$

Interestingly, we recover exactly the same minimal uncertainty as for standard Ramsey spectroscopy (42). This effect is illustrated in Fig. 2. The modulus of the frequency uncertainty $\left|\delta \omega_{0}\right|$ is plotted as a function of the duration of each single experiment $t$ for standard Ramsey spectroscopy with $n$ uncorrelated particles and for a maximally entangled state with $n$ particles. In the presence of decoherence both preparations reach the same optimal precision. This result can be intuitively understood by considering that maximally entangled states are much more fragile in the presence of decoherence: their decoherence time is reduced by a factor $n$ and therefore the duration of each single measurement $t$ has also to be reduced by the same amount. The previous conclusions hold whenever the total duration of the experiment exceeds the typical decoherence time. Hence, maximally entangled states are only advantageous for short term stabilizations.

Therefore, the conclusion we may draw so far is that the theoretical possibility of overcoming the shot noise limit by making use of maximally entangled states instead of uncorrelated preparations occurs only when the total duration of the experiment is comparable to the characteristic decoherence time. However, in long term experiments, as the ones required for the performance of a frequency standard, both preparations yield the same precision. Nevertheless, we have analyzed a particular method for performing precision spectroscopy and one may wonder whether the implementation of more general measurements could improve the final precision. Formulated in these terms, the problem addressed in precision spectroscopy (i.e. the optimal measurement of small atomic phase shifts) maps onto that of statistical distinguishability of nearby states, analyzed by Wootters [30] and generalized for the case of mixed states by Braunstein and Caves [31]. By finding measurements that optimally resolve neighbouring states, they have provided an upper bound for the precision in the estimation of a given variable that parametrizes a family of quantum states. In our case this variable is the detuning $\Delta$. Moreover, the optimal measurements always correspond to a set of orthogonal projectors in the $n$ ions' Hilbert space. It is worthwhile pointing out the generality of this result in the sense that it accounts for any possible joint measurement on the $n$ particles and any method of data analysis. When the Braunstein and Caves optimization procedure is applied [32]

- Uncorrelated or maximally entangled preparations of $n$ ions yield the same limit (42). In this sense, Ramsey spectroscopy is optimal when dealing with independent or maximally entangled preparations.

- The initial state preparation which leads to the best precision is of the form

$$
\left|\Psi_{n}\right\rangle=\sum_{k=0}^{\left\lfloor\frac{n}{2}\right\rfloor} a_{k}|k\rangle,
$$

where $|k\rangle$ denotes an equally weighted superposition of all states of $n$ ions which contain either a number $k$ or a number $n-k$ of excited states. By $\lfloor\cdots\rfloor$ we denote the integer part.

The optimum percentage improvement in the precision with respect to the limit (42) as a function of the number of ions $n$ is shown in Fig. 3 . The solid curve shows the improvement obtained by optimizing both the initial preparation and the final measurement using the algorithm of Braunstein and Caves. The dashed line exhibits the improvement obtained by optimizing only the initial preparation and performing the final measurement corresponding to Ramsey spectroscopy. The improvement obtained by optimising the measurement is rather small. It is still an open problem to study the asymptotic behaviour (for large values of $n$ ) of both curves, and in particular to understand whether the curve corresponding to Braunstein and Caves optimization saturates at the same value or higher than the Ramsey curve.

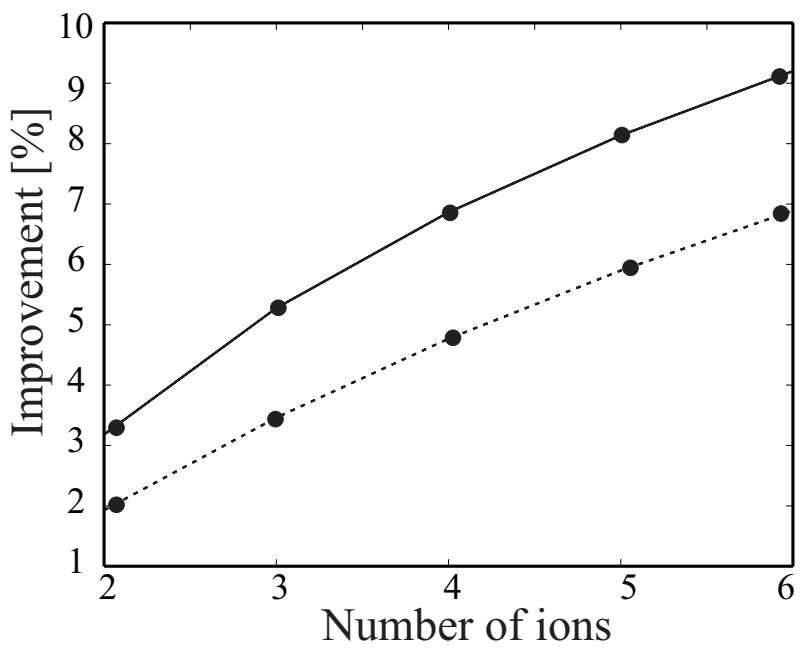



the initial preparation and application of the Braunstein and Caves algorithm for determining the optimal measurement. Dashed line: Optimized initial preparation and Ramsey type spectroscopy as the final measurement.

We will now briefly show a new method to improve the optimal precision (42) of frequency standards with uncorrelated particles in the presence of decoherence. Such a method is based on the use of a symmetrisation procedure [33], originally proposed as a technique to suppress errors in quantum computation. The key-point is to realize that during the free evolution period, in the absence of decoherence, the state of $n$ particles initially prepared in a state which is invariant under any permutation of the $n$ ions always lies in the symmetric subspace of the $2^{n}$-dimensional Hilbert space of the $n$ ions. By symmetric subspace we mean the subspace spanned by all states which are invariant under any permutation of the $n$ ions. A projection of the global state into the symmetric subspace would then yield a partial removal of events affected by environmental phase errors. Figure 4 shows the percentage precision improvement achievable with this technique for $n=2$. In this case a standard Ramsey scheme with initially uncorrelated ions has been considered and repeated symmetrisation steps are applied during the free evolution region. After each symmetrisation step the ions are kept only if the symmetrisation is successful, namely if the surviving state corresponds to the symmetric component of the state before symmetrisation. Otherwise the ions are discarded and reset to state $|0\rangle$ to start the scheme from the beginning. Although this reduces the number of experimental data available for statistics, Fig. 4 shows that it is a convenient strategy to improve the overall precision of the experiment. Notice that for frequent repetitions of symmetrisation during the free evolution period the improvement obtained over the shot noise (42) for uncorrelated particles is larger than the one achieved by optimising the initial preparation and the final measurement according to the Braunstein and Caves procedure (compare Fig. 4 with Fig. 3 at $n=2$ ).

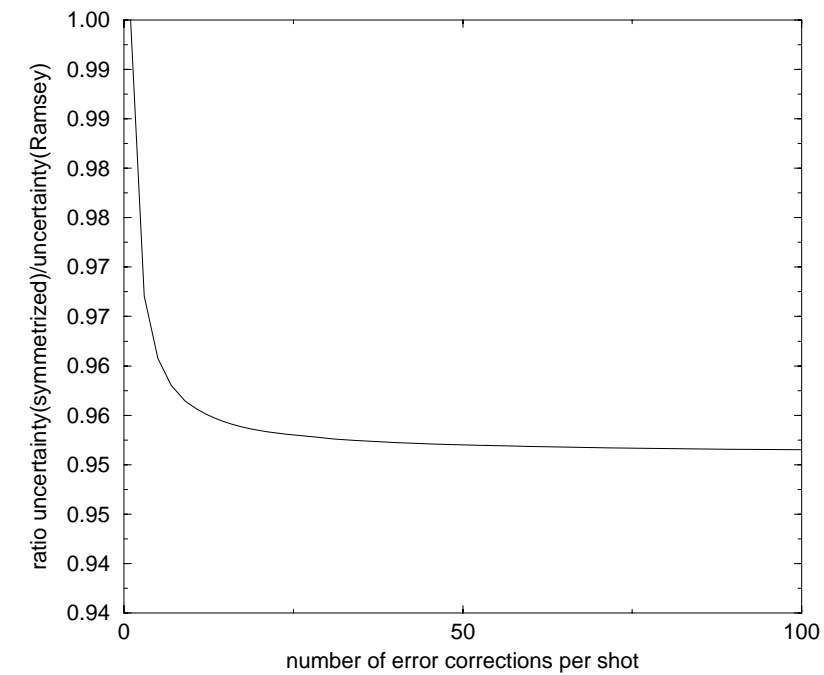

FIG. 7. Ratio of the uncertainty for initially uncorrelated particles in standard Ramsey spectroscopy with and without symmetrisation for $n=2$ as a function of the number of symmetrisation steps performed during the free evolution region.

The limits to the precision achievable with symmetrisation procedures for generic $n$ and a generic initial preparation of the state of the ions are still under investigation.

Acknowledgements. The authors would like to acknowledge fruitful discussions with J.I.Cirac and A. Ekert with whom part of the work presented here has been carried out. This work was supported in part by ElsagBailey, the UK Engineering and Physical Sciences Research Council (EPSRC), the European TMR Research Network ERBFMRXCT960066 and the European TMR Research Network ERBFMRXCT960087.

[1] J.S. Bell, Rev. Mod. Phys. 38, 447 (1966)

[2] T.M. Cover and J.A. Thomas, "Elements of Information Theory" (A Wiley-Interscience Publication, 1991).

[3] B. Schumacher, Phys. Rev. A 51, 2783 (1995).

[4] N. Gisin, Phys. Lett. A 210, 151, (1996), and references therein.

[5] K. Kraus, States, Effects, and Operations: Fundamental Notions of Quantum Theory (Springer Verlag, Berlin 1983)

[6] C. H. Bennett et al, Quantum Non-locality without entanglement, lanl e-print quant-ph/9804053.

[7] C.H Bennett, H.J. Bernstein, S. Popescu, and B. Schumacher, Phys. Rev. A 53, 2046 (1996); D. Deutsch, 
A. Ekert, R. Jozsa, C. Macchiavello, S. Popescu, and A. Sanpera Phys. Rev. Lett. 77, 2818 (1996).

[8] M. B. Plenio and V. Vedral, Teleportation, Entanglement and Thermodynamics in the Quantum World., to appear in Contemp. Phys., 1998.

[9] V. Vedral, M.B. Plenio, M.A. Rippin, and P.L. Knight, Phys. Rev. Lett. 78, 2275 (1997).

[10] M. Horodecki, P. Horodecki and R. Horodecki, Phys. Rev. Lett. 78, 574 (1997).

[11] M. Horodecki, P. Horodecki and R. Horodecki, Mixed state entanglement and distillation: is there a bound entanglement in nature?, lanl e-print quant-ph/9801069.

[12] V. Vedral and M. B. Plenio, Phys. Rev. A 57, 1619 (1998).

[13] T. Rockafeller, "Convex Analysis" (Princeton University Press, New Jersey, 1970).

[14] C.H. Bennett, D.P. DiVincenzo, J.A. Smolin, and W.K. Wootters, Phys. Rev. A 54, 3824 (1996).

[15] W.K. Wootters, Phys. Rev. Lett. 80, 2245 (1998).

[16] V. Vedral, M.B. Plenio, K. Jacobs, and P.L. Knight, Phys. Rev. A 56, 4452 (1997).

[17] F. Hiai and D. Petz, Comm. Math. Phys. 143, 99 (1991).

[18] M. Ohya, Rep. Math. Phys. 27, 19 (1989).

[19] M.J. Donald, Comm. Math. Phys. 105, 13 (1986); M.J. Donald, Math. Proc. Camb. Phil. Soc., 101, 363 (1987).

[20] A. Wehrl, Rev. Mod. Phys. 50, 221 (1978).

[21] C. A. Fuchs, "Distinguishability and Accessible Information in Quantum Theory" $\mathrm{PhD}$ thesis, The University of New Mexico, Albuquerque, NM, 1996 (lanl e-print quant$\mathrm{ph} / 9601020)$.

[22] M. Hayashi, "Asymptotic Attainment for Quantum Relative Entropy", lanl e-print server: quant-ph/9704040 (1997).

[23] M. J. Donald, Found. Phys. 22, 1111 (1992).

[24] E. Rains, Entanglement purification via separable superoperators, lanl e-print quant-ph/9707002.

[25] N. Ramsey, Molecular Beams, Oxford 19...

[26] W. H. Itano et al., Phys. Rev. A47, 3554 (1993).

[27] W. J. Wineland et al., Phys. Rev. A46, R6797 (1992).

[28] D. J. Wineland, J.J. Bollinger, W.M. Itano, and D.J. Heinzen, Phys. Rev. A50, 67 (1994).

[29] C. W. Gardiner, Quantum Noise (Springer-Verlag, Berlin 1991).

[30] W. K. Wootters, Phys. Rev. D23, 357 (1981)

[31] S. L. Braunstein and C. Caves, Phys. Rev. Lett. 72, 3439 (1994)

[32] S. F. Huelga, C. Macchiavello, T. Pellizari, A. K. Ekert, M. B. Plenio and J. I. Cirac, Phys. Rev. Lett. 79, 3865 (1997)

[33] A. Barenco, A. Berthiaume, D. Deutsch, A. Ekert, R. Jozsa and C. Macchiavello, SIAM J. Comput. 26, 1541 (1997). 